\title{
Conserved Genoarchitecture of the Basal Hypothalamus in Zebrafish Embryos
}

\author{
Theresa Schredelseker ${ }^{1,2 *}$ and Wolfgang Driever ${ }^{1,2 *}$ \\ ${ }^{1}$ Developmental Biology, Institute Biology I, Faculty of Biology, University of Freiburg, Freiburg, Germany, ${ }^{2} \mathrm{CIBSS}$ \\ and BIOSS - Centres for Biological Signalling Studies, University of Freiburg, Freiburg, Germany
}

OPEN ACCESS

Edited by:

Luis Puelles,

University of Murcia, Spain

Reviewed by:

José L. E. Ferran,

University of Murcia, Spain Isabel Rodríguez-Moldes,

University of Santiago

de Compostela, Spain

*Correspondence:

Theresa Schredelseker

schredet@tcd.ie

Wolfgang Driever

driever@biologie.uni-freiburg.de

Received: 05 October 2019

Accepted: 20 January 2020

Published: 06 February 2020

Citation:

Schredelseker $T$ and Driever W (2020) Conserved Genoarchitecture of the Basal Hypothalamus

in Zebrafish Embryos.

Front. Neuroanat. 14:3.

doi: 10.3389/fnana.2020.00003
Analyses of genoarchitecture recently stimulated substantial revisions of anatomical models for the developing hypothalamus in mammalian and other vertebrate systems. The prosomeric model proposes the hypothalamus to be derived from the secondary prosencephalon, and to consist of alar and basal regions. The basal hypothalamus can further be subdivided into tuberal and mamillary regions, each with distinct subregions. Albeit being a widely used model system for neurodevelopmental studies, no detailed genoarchitectural maps exist for the zebrafish (Danio rerio) hypothalamus. Here, we compare expression domains of zebrafish genes, including arxa, shha, otpa, is/1, Ihx5, $n k \times 2.1, n k \times 2.2 a$, pax6, and $d / x 5 a$, the orthologs of which delimit specific subregions within the murine basal hypothalamus. We develop the highly conserved brain-specific homeobox (bsx) gene as a novel marker for genoarchitectural analysis of hypothalamic regions. Our comparison of gene expression patterns reveals that the genoarchitecture of the basal hypothalamus in zebrafish embryos 48 hours post fertilization is highly similar to mouse embryos at E13.5. We found the tuberal hypothalamus in zebrafish embryos to be relatively large and to comprise previously ill-defined regions around the posterior hypothalamic recess. The mamillary hypothalamus is smaller and concentrates to rather medial areas in proximity to the anterior end of the neural tube floor plate. Within the basal hypothalamus we identified longitudinal and transverse tuberal and mamillary subregions topologically equivalent to those previously described in other vertebrates. However, the hypothalamic diencephalic boundary region and the posterior tuberculum still provide a challenge. We applied the updated prosomeric model to the developing zebrafish hypothalamus to facilitate cross-species comparisons. Accordingly, we applied the mammalian nomenclature of hypothalamic organization to zebrafish and propose it to replace some controversial previous nomenclature.

Keywords: prosomeric model, bsx brain-specific homeobox, zebrafish brain development, genoarchitecture, patterning, progenitor domains, tuberal hypothalamus, mamillary region

\section{INTRODUCTION}

The hypothalamus comprises a group of important neuroendocrine and neuromodulatory nuclei. Its organization within the forebrain has been investigated extensively (Puelles et al., 2012; Xie and Dorsky, 2017). Gene expression domains proved a valuable tool in comparative neuroanatomy, since the patterns they produce often remain topologically homologous through 
evolution, despite considerable shifts occurring in some species. Their analysis thus allows to generate genoarchitectonic maps which help in understanding both developmental and evolutionary relationships between forebrain regions (Puelles and Ferran, 2012).

The caudal part of the primary prosencephalon (forebrain) will give rise to the diencephalon, which has been suggested to be subdivided into transverse units called prosomers. The rostral part of the prosencephalon is considered to represent a complex protosegment, termed secondary prosencephalon (Puelles and Rubenstein, 2003), and will give rise to both the telencephalon and the hypothalamus.

In contrast to columnar models of brain architecture, which consider the whole hypothalamus to derive from the basal plate (reviewed in Bedont et al., 2015), the prosomeric model proposes a subdivision of the hypothalamus into both alar and basal regions. According to the most recently updated prosomeric model, the hypothalamus can be subdivided from caudal to rostral into peduncular, terminal and acroterminal regions (Puelles et al., 2012; Ferran et al., 2015). The acroterminal region was defined as derivative of the rostral-most neural tube, and fate-mapping experiments revealed its dorsal derivatives to correspond to the anterior commissure region and optic chiasm, while the ventral derivatives include neurohypophysis, arcuate

\footnotetext{
Abbreviations: A, anterior (colinear to neuraxis); $\mathrm{A}^{*}$, anterior (colinear to body axis); ABas, anterobasal region; AC, anterior commissure; aHyp, alar hypothalamus; ARC, arcuate nucleus; bsx, brain-specific homeobox; cHyp, "caudal hypothalamus" (1); D, dorsal (colinear to neuraxis); $\mathrm{D}^{*}$, dorsal (colinear to body axis); DC, diencephalic cluster (of dopaminergic neurons) (2); DM, dorsomedial nucleus region; DT, "dorsal thalamus" (1); Epi, epithalamus; Hab, habenula; hpf, hours post fertilization; Hyp, hypothalamus; iHyp, "intermediate hypothalamus" (1); LR, lateral recess; MA, mamillary domain (sensu stricto); Mam, mamillary regions (sensu lato); MM, mamillary region (columnar model) (3); NPO, neurosecretory preoptic region; OC, optic commissure; OR, optic recess; ORR, optic recess region; $\mathrm{P}$, posterior (colinear to neuraxis); $\mathrm{P}^{*}$, posterior (colinear to body axis); PC, posterior commissure; pHyp, "posterior Hypothalamus" (1); pin, pineal gland; pit, pituitary gland; PM, perimamillary domain; pp, parapineal organ; PreM, premamillary region (3); PRM, periretromamillary domain; PR, posterior (hypothalamic) recess; PRR, posterior recess region; PTar, "posterior tuberculum, anterior rostral" dopaminergic neurons (1); PTd, "posterior tuberculum, dorsal part" (1); pTh, prethalamus; PTv, "posterior tuberculum, ventral part" (1); p3a, prosomer 3, alar part; $\mathrm{p} 3 \mathrm{~b}$, prosomer 3, basal part; RM, retromamillary domain; $\mathrm{RTuD}$, retrotuberal domain, dorsal part; RTuI, retrotuberal domain, intermediate part; RTuV, retrotuberal domain, ventral part; SMM, supramammillary nucleus region (3); SPV, supraopto-paraventricular region; Tel, telencephalon; TT, tuberomamillary terminal (3); Tub, tuberal regions (in sensu lato); $\mathrm{TuD}$, tuberal domain, dorsal part; TuI, tuberal domain, intermediate part; TuV, tuberal domain, ventral part; $\mathrm{V}$, ventral (colinear to neuraxis); $\mathrm{V}^{*}$, ventral (colinear to body axis); Ve, ventricle; vHyp, "ventral hypothalamus" (1); VMH, ventromedial hypothalamic nucleus region; VT "ventral thalamus" (1).

(1) Quotation marks indicate anatomical designations which refer to the actual position relative to the whole zebrafish embryo body axis. We propose that those should be replaced by terms defined within the updated prosomeric model framework, taking into account the strong cephalic flexure and referring to the neuraxis.

(2) Note that within the updated prosomeric model framework, which considers the hypothalamus as derivative of the secondary prosencephalon and not part of the diencephalon proper, the classical term "diencephalic cluster" (Rink and Wullimann, 2002) is misleading since at least some of those dopaminergic cell clusters are located outside the diencephalon proper.

(3) These terms are not used throughout the manuscript with the sole exception of Supplementary Figure S2 in which a model of the basal hypothalamus based on
} the columnar model of brain architecture is presented. nucleus and finally the median mamillary area (discussed in Puelles et al., 2012).

Within the hypothalamus four longitudinal domains can be distinguished from dorsal to ventral: paraventricular and subparaventricular in the alar plate and tuberal and mamillary in the basal plate (Puelles et al., 2012; Puelles and Rubenstein, 2015). Within the basal hypothalamus, the mamillary regions can further be divided into a more ventral area, consisting of the peduncular retromamillary domain (RM) and the terminal mamillary domain (sensu stricto, MA) and a more dorsal region, consisting of the peduncular periretromamillary domain (PRM) and the terminal perimamillary domain (PM). Similarly, the tuberal hypothalamus is subdivided into ventral, intermediate and dorsal components, usually abbreviated as TuV, TuI, $\mathrm{TuD}$ and $\mathrm{RTuV}, \mathrm{RTuI}, \mathrm{RTuD}$ for terminal and peduncular subdomains, respectively (Puelles and Rubenstein, 2015).

Most of the updated prosomeric model of the hypothalamus was established based on gene expression patterns in mouse embryonic brain development (Morales-Delgado et al., 2011; Díaz et al., 2014; Morales-Delgado et al., 2014; Ferran et al., 2015). However, being conceptualized as a cross-species vertebrate model, it has also been applied with species-specific adaptations to frogs (Dominguez et al., 2013, 2014; González et al., 2017), reptiles (Moreno et al., 2012, 2017; Dominguez et al., 2015), and catsharks (Santos-Duran et al., 2015, 2018; SantosDurán et al., 2016) recently. Even though zebrafish represent a widely used model organism for developmental neurobiology, to our knowledge no study as of yet attempted to apply the updated model to zebrafish. In contrast, for the zebrafish larval hypothalamus often a heuristic subdivision into rostral, intermediate and caudal hypothalamus is used (Wullimann et al., 1996; Manoli and Driever, 2014; Biran et al., 2015; Mueller and Wullimann, 2016; Muthu et al., 2016). These anatomical terms of location, however, can be misleading as they refer to actual positions in the embryo and not to their presumptive embryological and genoarchitectonic origin in relation to the neural tube. Since the zebrafish neural tube, however, forms a pronounced cephalic flexure, those two coordinate systems are highly incongruent.

Recent anatomical analyses of the hypothalamus focused on the alar regions (Herget et al., 2014; Nagpal et al., 2018). Even more recently, a novel anatomical entity, the optic recess region (ORR) has been proposed to render the concept of an alar hypothalamus obsolete (Affaticati et al., 2015; Yamamoto et al., 2017; Alié et al., 2018). Very little attention has been paid to the teleost basal hypothalamus for which homology with its mammalian counterpart has been considered to be modest (Vernier, 2017). In contrast, here we apply the updated prosomeric model (Puelles et al., 2012; Puelles and Rubenstein, 2015) to the developing zebrafish basal hypothalamus and find extensive homology.

In our work, we follow the parsimony principle, i.e., demarcate regions assuming the highest grade of homology, and aim to describe a genoarchitectural organization of the zebrafish embryonic hypothalamus postulating as few as possible evolutionary changes. We selected genes whose expression domains in model organisms for other vertebrate classes have 
been shown to represent defined progenitor domains within the embryonic basal hypothalamus, and analyzed expression of those genes in the zebrafish brain 48 hours post fertilization (hpf), a stage at which the cephalic flexure is fully formed and the major regionalization of the brain is essentially completed (Mueller and Wullimann, 2016).

We were able to identify both tuberal and mamillary regions and therein several subdomains which topologically correspond to those of the updated prosomeric model. When we detected significant differences in gene expression domains between optical sections closer or further away from the midline, we mostly labeled those as "midsagittal," "intermediate sagittal" or "parasagittal" (Supplementary Figure S1), rather than "periventricular" or "mantle," to account for ventricular recesses and to avoid confusion with neurogenic zones. If not specified in the image panels, intermediate sagittal sections (Supplementary Figure S1) are shown.

Alongside our genoarchitectural analysis of the basal hypothalamus we characterized the expression pattern of the brain-specific homeobox (bsx) gene in detail. The expression pattern of this homeobox transcription factor has previously been described in mice (Cremona et al., 2004). This work was, however, prior to the prosomeric model being well-established, and comprehensive reviews of the updated prosomeric model do not discuss Bsx (Puelles et al., 2012). In this study we demonstrate the hypothalamic regionalization to be highly conserved throughout evolution and propose that expression of the conserved $b s x$ transcription factor might function as a valuable marker for several distinct subdomains therein.

\section{MATERIALS AND METHODS}

\section{Animal Strains and Maintenance}

Wildtype ABTL zebrafish were kept at $28.5^{\circ} \mathrm{C}$ on a $14 \mathrm{~h}$ light $/ 10 \mathrm{~h}$ dark cycle and natural mating was allowed in breeding traps. Embryos were kept in $3 \mathrm{~g} / \mathrm{l}$ Red Sea salt (Red Sea) at $28.5^{\circ} \mathrm{C}$ and staged according to Kimmel et al. (1995). Starting from $8 \mathrm{hpf}$, $0.2 \mathrm{mM}$ N-phenylthiourea (Sigma-Aldrich) was used to prevent pigmentation. All experiments were carried out in accordance with the German Animal Welfare Act.

\section{Cloning of cDNA Fragments for Probe Synthesis}

A list of all transcripts which were used as targets for in situ hybridization probes is given in Table 1. For all probes which have been described previously, references are given in Supplementary Table S1. Templates for all other probes were PCR amplified from cDNA and subcloned into pCRIITOPO plasmid (Invitrogen) as has been described before (Schredelseker and Driever, 2018). Primer sequences are shown in Supplementary Table S1.

\section{Whole-Mount in situ Hybridization}

At 48, 72 or 96 hpf manually dechorionated embryos were fixed in $4 \% \mathrm{PFA} / \mathrm{PBST}\left(137 \mathrm{mM} \mathrm{NaCl}, 2.7 \mathrm{mM} \mathrm{KCl}, 10 \mathrm{mM} \mathrm{Na}_{2} \mathrm{HPO}_{4}\right.$,
$1.8 \mathrm{mM} \mathrm{KH}_{2} \mathrm{PO}_{4}, 0.1 \%$ Tween 20$)$ overnight at $4^{\circ} \mathrm{C}$ before being dehydrated stepwise (25\% MeOH/PBST, 50\% MeOH/PBST, 75\% $\mathrm{MeOH} / \mathrm{PBST}, 5 \mathrm{~min}$ each) and stored in $\mathrm{MeOH}$ at $-20^{\circ} \mathrm{C}$ before being used for whole-mount in situ hybridization. Doublefluorescent whole-mount in situ hybridization was carried out as previously described (Filippi et al., 2007; Ronneberger et al., 2012). In very few reactions we observed TSA cross-reactions leading to weak fluorescent labeling of individual probes in not only one but both channels ( $b s x$ in Figure 5F, $n k x 2.1$ in Supplementary Figure S5C' and $b s x$ in Supplementary Figure S5D). However, cross-reaction is so weak that it does not obscure the results of the expression analysis.

\section{Imaging and Figure Preparation}

All recordings were made on a Zeiss LSM 880 using an LD-LCI Plan Apochromat 25x/0.8 objective. The Zeiss ZEN Black software was used to make linear adjustments of levels through histogram clipping and to generate maximum intensity projections of selected focal planes. Figures were assembled using Adobe Photoshop CS5 or CS6. Full z-stacks are available upon request.

\section{RESULTS}

\section{Subdivision of the Basal Hypothalamus in Zebrafish Larvae Into Mamillary and Tuberal Regions}

To better understand the cephalic flexure and anteriorposterior organization of the forebrain in zebrafish, we aimed to identify the anterior end of the floor plate in $48 \mathrm{hpf}$ embryos. Given that the floor plate in mouse embryos has been shown to express Shh and Arx (Cho et al., 2014), we performed double fluorescent in situ hybridization using arxa and shha probes (Figures 1A-B') neuroanatomical analyses and fate mapping experiments carried out by others (discussed in Puelles et al., 2012), we hypothesized the region around the rostral most end of the shha $a^{+}$arxa $^{+}$floor plate (Figures $\mathbf{1 A}^{\prime \prime}, \mathbf{B}^{\prime \prime}$ and Supplementary Figures S2A-A", arrowheads) to develop into mamillary regions of the hypothalamus. Beyond the floor plate, arxa and shha in the secondary prosencephalon are expressed mostly in a mutually exclusive pattern, marking the alar-basal boundary (Puelles et al., 2012; Figures 1A-A' $\mathbf{A}^{\prime \prime}$ ). While shha expression in the basal hypothalamus is mainly restricted to medial (i.e., midsagittal or periventricular) regions, arxa expression forms a more laterally located band within the basal hypothalamus (Figures 1B-B" and Supplementary Figures S2E-E ${ }^{\prime \prime}$ ). Based on Arx expression in mouse embryonic development (Puelles et al., 2012), we hypothesized this band to correspond to the ventralmost retrotuberal and tuberal areas $(\mathrm{TuV} / \mathrm{RTuV}$, Figures $\left.\mathbf{1 B}-\mathbf{B}^{\prime \prime}, \mathbf{D}-\mathbf{D}^{\prime \prime}\right)$. We supported this interpretation through the observation that this $\operatorname{arxa}^{+}$domain also expresses lhx6, defining the TuV/RTuV region (Puelles et al., 2012; Supplementary Figures S2B-B' ${ }^{\prime \prime}$ ). This $\operatorname{arxa}^{+}$, $l h x \sigma^{+}$band is called tuberomamillary terminal by proponents of the columnar 
TABLE 1 | All genes for which RNA antisense probes have been used for in situ hybridization.

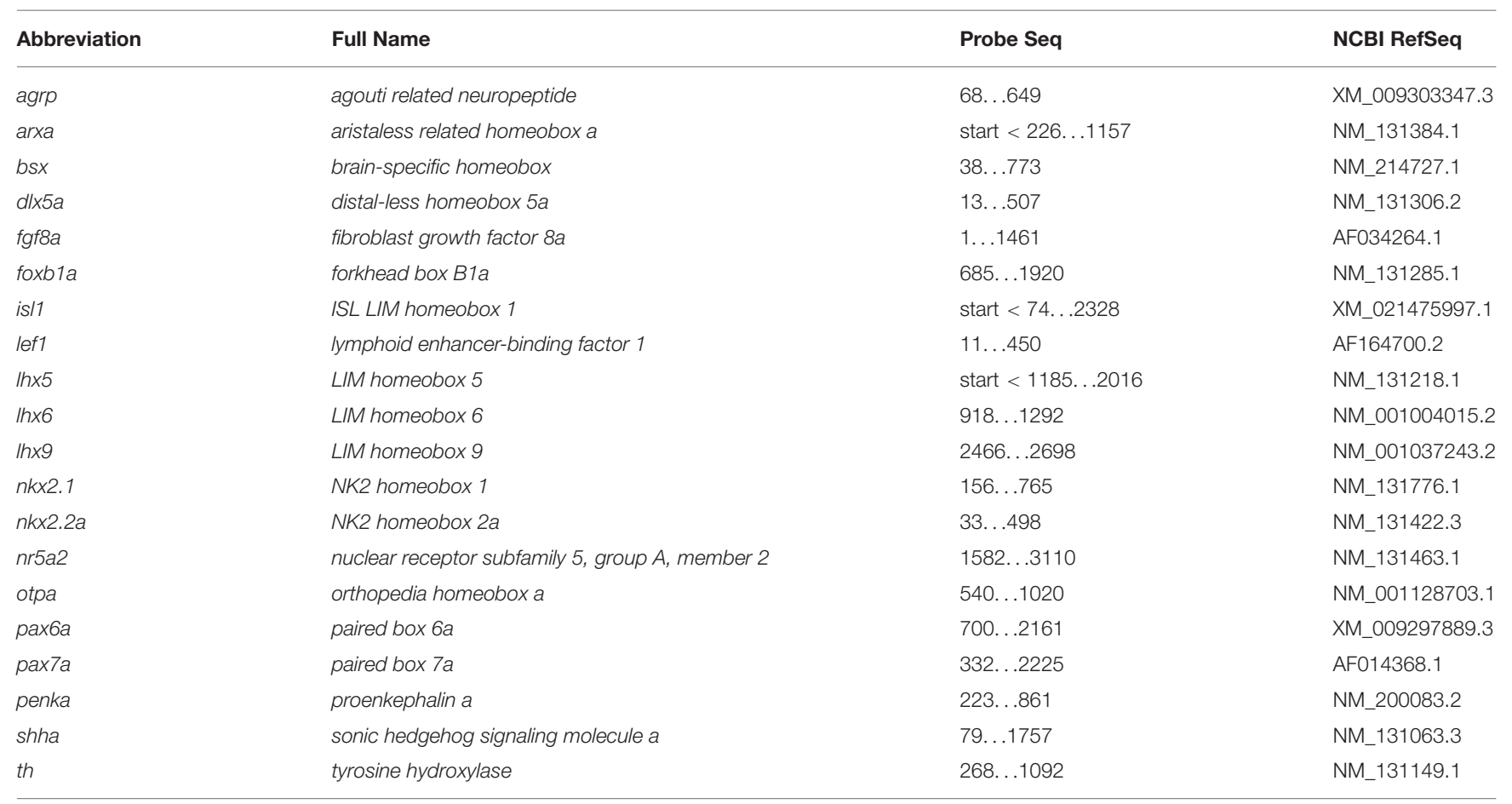

Third column indicates the sequence range used for probe generation. Numbering refers to the reference sequence indicated in fourth column. References for published probes and primer sequences for probes which were cloned in this study can be found in Supplementary Table S1.

model (Shimogori et al., 2010; Supplementary Figure S2H) and in the updated prosomeric model represents the border between the tuberal and mamillary regions (Puelles et al., 2012; Supplementary Figure S2I). shha and arxa expression reveal that parts of an area surrounding the rostral end of the neural tube, which often has been referred to as "ventral posterior tuberculum region" ("PTv"; Mueller and Wullimann, 2016), actually correspond to mamillary hypothalamus (Figure $\mathbf{1 H}$ and Supplementary Figure S2J). Consequently, we considered a quite large area on the other side of the $\operatorname{arxa}^{+}, \operatorname{lh} \times 6^{+}$band to be located embryologically dorsal to mamillary regions, and thus representing tuberal hypothalamus (Figure $\mathbf{1 H}$ and Supplementary Figure S2J). This area has previously been labeled "caudal" or "posterior" hypothalamus (Wolf and Ryu, 2013; Xie and Dorsky, 2017; Figure 1G). We confirmed the tuberal nature of this area through the expression of isll, which is moderate in medial regions (Figures $\mathbf{1} \mathbf{C}-\mathbf{C}^{\prime \prime}$ ), but more pronounced and expanded toward the end of the floor plate in more lateral (i.e., parasagittal, mantle) areas (Figures $\mathbf{1 D}-\mathbf{D}^{\prime \prime}$ ). We concluded that the isll negative mamillary regions are larger medially and thinner laterally (Figures $\mathbf{1 H}^{\prime}-\mathbf{H}^{\prime \prime}$ ). Similar observations have been reported previously for mouse (Puelles et al., 2012). We found otpa in the zebrafish basal hypothalamus to be expressed in both tuberal (Figures 1E-E', arrow in Figure $1 \mathbf{E}^{\prime}$ and Supplementary Figures $\left.\mathbf{S 2 C}-\mathbf{D}^{\prime \prime}\right)$ and mamillary regions (Figures 1E- $\mathbf{E}^{\prime \prime}$, arrowhead in Figure $1 \mathbf{E}^{\prime}$ and Supplementary Figures $\mathbf{S 2 C}-\mathbf{D}^{\prime \prime}$ ). This is in line with murine Otp being expressed within the PM/PRM band as well as in dorsal tuberal regions (TuD; Morales-Delgado et al., 2014; Ferran et al., 2015). Again, we observed the mamillary otpa expression domain to be larger midsagittally and the isl1 expressing tuberal regions to surround this domain at parasagittal levels (Figures 1E-F" and Supplementary Figures S2F-G'). We will discuss the organization of the basal hypothalamus along the radial axis in more details later. With respect to the rostro-caudal and dorso-ventral axis, we will use the terms anterior, posterior, dorsal and ventral related to the neuraxis, unless indicated otherwise (Figure $\mathbf{1 H}$ ). We propose a subdivision of the embryonic zebrafish basal hypothalamus into tuberal and mamillary regions (Figure $\mathbf{1 H}$ ) based on conserved genoarchitecture to replace previous models of hypothalamus organization into "anterior" or "rostral," "intermediate" or "ventral" and "posterior" or "caudal" zones (Figure 1G).

\section{bsx Is Expressed in Both Tuberal and Mamillary Regions of the Basal Hypothalamus}

The $b s x$ transcription factor is expressed in several domains within the secondary prosencephalon in mouse and zebrafish (Cremona et al., 2004; Schredelseker and Driever, 2018). Here, comparing $b s x$ expression with arxa as a marker for the alar territories, we found that most $b s x$ expression in the ventral

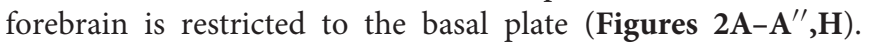
However, a small $b s x$ expression domain, located right at the alar-basal boundary, appears to be largely surrounded 

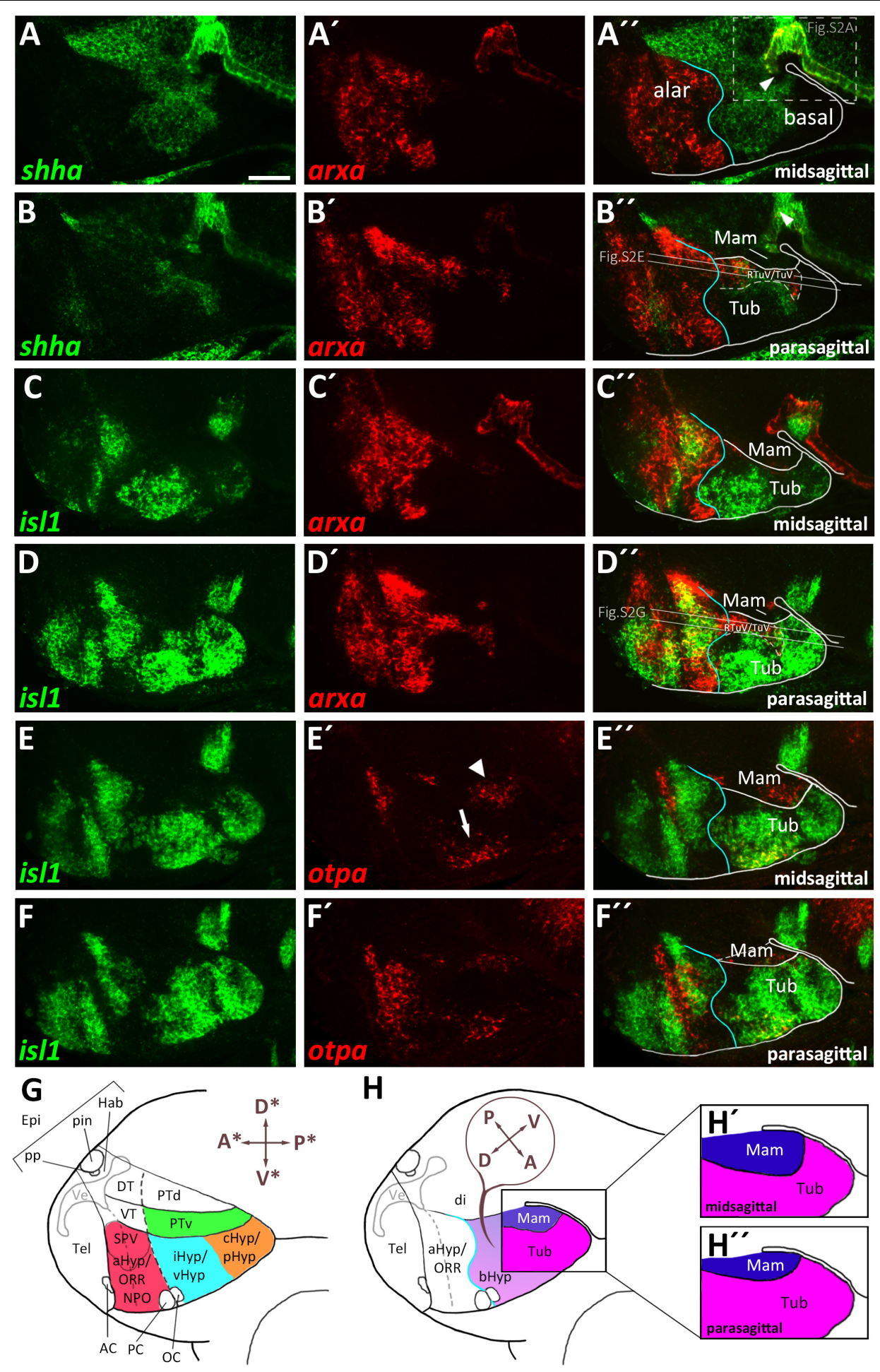

FIGURE 1 | Gene expression domains demarcate alar and basal territories within the secondary prosencephalon as well as tuberal and mamillary subregions within the basal hypothalamus. (A-F') Sagittal optical sections of zebrafish embryos $48 \mathrm{hpf}$ at medial, ventricular or more lateral, mantle positions as indicated, stained by double-fluorescent whole-mount in situ hybridization using probes as indicated. (G) Integration of several prevalent anatomical models of the zebrafish embryonic forebrain referencing orientation colinear to the body axis (Wullimann et al., 1996; Manoli and Driever, 2014; Biran et al., 2015; Mueller and Wullimann, 2016; Muthu et al., 2016). (H) Proposed model of hypothalamus subdivision into mamillary and tuberal areas at ventricular $\left(\mathbf{H}^{\prime}\right)$ or mantle zones $\left(\mathbf{H}^{\prime \prime}\right)$ with anatomic directions colinear to the neuraxis. Dashed box in $\left(\mathbf{A}^{\prime \prime}\right)$ indicates area which is shown in higher magnification in Supplementary Figure S2A. Arrowhead in $\left(\mathbf{A}^{\prime \prime}\right)$ indicates the rostral border of the floor plate. The alar-basal boundary is shown in cyan. Maximum intensity projections of $10\left(\mathbf{A}-\mathbf{A}^{\prime \prime}\right), 15\left(\mathbf{C}-\mathbf{C}^{\prime \prime}\right), 25\left(\mathbf{B}-\mathbf{B}^{\prime \prime}, \mathbf{D}-\mathbf{D}^{\prime \prime}, \mathbf{F}-\mathbf{F}^{\prime \prime}\right)$, or 35 (E-E') $1 \mu \mathrm{m}$ confocal planes. Abbreviations see list. Scale bar $50 \mu \mathrm{m}$. 
by arxa expression, albeit being arxa negative (Figure $\mathbf{2} \mathbf{A}^{\prime \prime}$, arrowhead). We observed a similar situation when we compared $b s x$ expression in this area to shha expression, marking basal regions (Puelles et al., 2012): bsx expressing cells appeared to be located directly adjacent but not within the shha expression area (Figures $\mathbf{5 B}-\mathbf{B}^{\prime \prime}$ ). shha expression is restricted to periventricular zones, while $b s x$ is expressed mainly in mantle layers. Therefore, it was difficult to determine if this $b s x$ domain belongs to the alar or basal plate. Next, we aimed to resolve if this domain is located within the hypothalamus or within the diencephalon. As a marker for the basal part of the hypothalamus abutting prosomer 3 (p3b) we used pax7a (Moreno et al., 2014), the expression of which, however, has been described to reach into the hypothalamus during some stages of amphibian development (Bandín Carazo et al., 2013; Joven et al., 2013). We detected adjacent expression of pax7a and $b s x$ (Figures 2E-E' ${ }^{\prime \prime}$ ). Co-staining of $b s x$ and pax $6 a$, the latter having been described to be expressed in the alar part of prosomer 3 (p3a; Martinez-Ferre and Martinez, 2012; Moreno et al., 2014), also revealed adjacent expression domains (Figures $2 \mathbf{D}-\mathbf{D}^{\prime \prime}$ ) rather than overlapping ones. Co-staining pax6a and pax7a confirmed existence of a domain located right at both, the border between alar and basal plate, and the border between prosomer 3 and hypothalamus (Figures 2F-G', arrowheads in Figures $2 \mathbf{F}^{\prime \prime}, \mathbf{G}$ ).

We found that directly lateral to the $b s x$ expressing domain in this area, th expressing dopaminergic neurons are located (Supplementary Figures S3D-E' ${ }^{\prime \prime}$ ). Those th expressing cells, previously referred to as the diencephalic cluster 2 (DC2), have been characterized to be located within the "PTv" (Vernier and Wullimann, 2009) and based on its relative position therein have recently been referred to as "anterior rostral posterior tuberculum" dopaminergic cells ("PTar"; Reinig et al., 2017). We thus operationally associated the $b s x$ expression in this region with the "PTv."

Expression of $b s x$ on both sides of the $\operatorname{arxa} a^{+} / \operatorname{lh} x 6^{+}$ $\mathrm{RTuV} / \mathrm{TuV}$ band (Figures 2A-A' and Supplementary Figures $\mathbf{S} 3 \mathbf{A}-\mathbf{A}^{\prime \prime}$ ) indicated that $b s x$ is expressed in both mamillary and tuberal regions. We confirmed $b s x$ expression in tuberal regions through a co-staining with isl 1 , which, in line with data from mice (Puelles et al., 2012), we found to be expressed broadly within the tuberal region, therein partly colocalizing with $b s x$ (Figures $\mathbf{2 B}-\mathbf{B}^{\prime \prime}, \mathbf{H}, \mathbf{I}$ ). In line with our previous results (Figures $\mathbf{1 C}-\mathbf{F}^{\prime \prime}$ ), we found the isl 1 expression domain to be expanded in parasagittal sections partially overgrowing the mamillary region laterally (Supplementary Figures S3B-C $\mathbf{C}^{\prime \prime}$ ). In the area containing "PTar" dopaminergic neurons, as well as in mamillary regions we found $b s x$ expression to overlap with otpa expression (Figures $\mathbf{2 C}-\mathbf{C}^{\prime \prime}, \mathbf{H}, \mathbf{I}$ ). In the tuberal hypothalamus we also found $b s x$ to colocalize with otpa, but additionally to be expressed in otpa negative tuberal areas (Figures $2 \mathbf{C}-\mathbf{C}^{\prime \prime}, \mathbf{H}, \mathbf{I}$ ). We will discuss these and other tuberal subregions in more detail in another section. We conclude that similarly to what has been described in mouse (Cremona et al., 2004), bs $x$ is expressed in both tuberal and mamillary regions within the basal hypothalamus suggesting that $b s x$ expression is highly conserved in the secondary prosencephalon.

\section{The Mamillary Region Can Be Subdivided Into Four Territories}

To identify mamillary subregions based on gene expression patterns described in other model organisms, we analyzed the expression domains of shha, nkx2.1, otpa, foxb1a, lhx5 and $b s x$. To confirm that otpa is expressed in both the peduncular PRM and terminal PM, we analyzed its expression relative to shha, which in mouse labels the peduncular PRM and RM domains but not the terminal mamillary regions, PM and MA (Puelles et al., 2012). In line, we found otpa and shha expression to overlap in a posterior, dorsal mamillary domain which we assume to correspond to PRM (Figures $\mathbf{3 A}-\mathbf{A}^{\prime \prime}$ ). We found that an area closer toward the floor plate, thus embryologically ventral, expresses shha but not otpa (Figures $\mathbf{3} \mathbf{A}-\mathbf{A}^{\prime \prime}$ ) and reasoned that this domain should be considered RM. Consistent with data from mouse (Puelles et al., 2012), the mamillary otpa expression domain extends anteriorly (in relation to the neuraxis) into a shha negative area, presumably representing PM (Figures $\mathbf{3} \mathbf{A}-\mathbf{A}^{\prime \prime}$ ).

To confirm this model, we compared expression of shha to the expression of $n k x 2.1$ which in mice is expressed in all mamillary regions except RM (Puelles et al., 2012). This allowed us to reinforce RM as a distinct domain expressing shha but not $n k x 2.1$ and PRM as expressing both shha and $n k x 2.1$ (Figures 3B-B' ${ }^{\prime \prime}$ ). We further concluded that the $n k x 2.1^{+}, s h h a^{-}$areas encompass the terminal mamillary domains PM and MA (Figures 3B-B' ${ }^{\prime \prime}$ ). As we previously showed $b s x$ expression to perfectly overlap with otpa expression in the mamillary regions (Figures $\mathbf{2} \mathbf{C}-\mathbf{C}^{\prime \prime}$ ), we used $b s x$ and otpa interchangeably as markers for the PRM/PM band. Ihx5 has recently been described in catshark as being expressed in PRM, PM and MA but not in RM (Santos-Duran et al., 2018). Co-staining of $l h x 5$ and $b s x$ suggested that this expression pattern is conserved in zebrafish and that $b s x$ is expressed in PRM as well as PM but not in MA (Figures $3 \mathrm{C}-\mathbf{C}^{\prime \prime}$ ). To support this hypothesis, we used foxbla as a marker for the MA region (Zhao et al., 2008; Puelles et al., 2012) and found expression close to the end of the floor plate in an area which does not express $b s x$ (Figures 3D-D' ${ }^{\prime \prime}$ ). We concluded that in the embryonic zebrafish hypothalamus, mamillary regions can be defined and divided into four subdomains corresponding to those previously described in mammals (Figure 3E; Puelles et al., 2012).

\section{The Mamillary Domain Is Larger in Medial Regions and Flanked by Both Perimamillary and Tuberal Domains in Lateral Regions}

The model which we unfolded in the previous section shows the spatial organization of the mamillary subregions within medial regions of the basal hypothalamus, i.e., those regions that lie close to the ventricle. We noted, however, that the borders shown therein are shifted as we analyzed lateral regions in parasagittal optical section. In those regions further away from the midline which do not express shha (Supplementary Figures S4A-B'), we found expression of markers indicative for the PRM/PM band, such as otpa (Supplementary Figures S4A, $\mathbf{A}^{\prime \prime}$ ), lhx5 and $b s x$ (Supplementary Figures $\mathbf{S} 4 \mathbf{C}-\mathbf{C}^{\prime \prime}$ ) to reach closer 

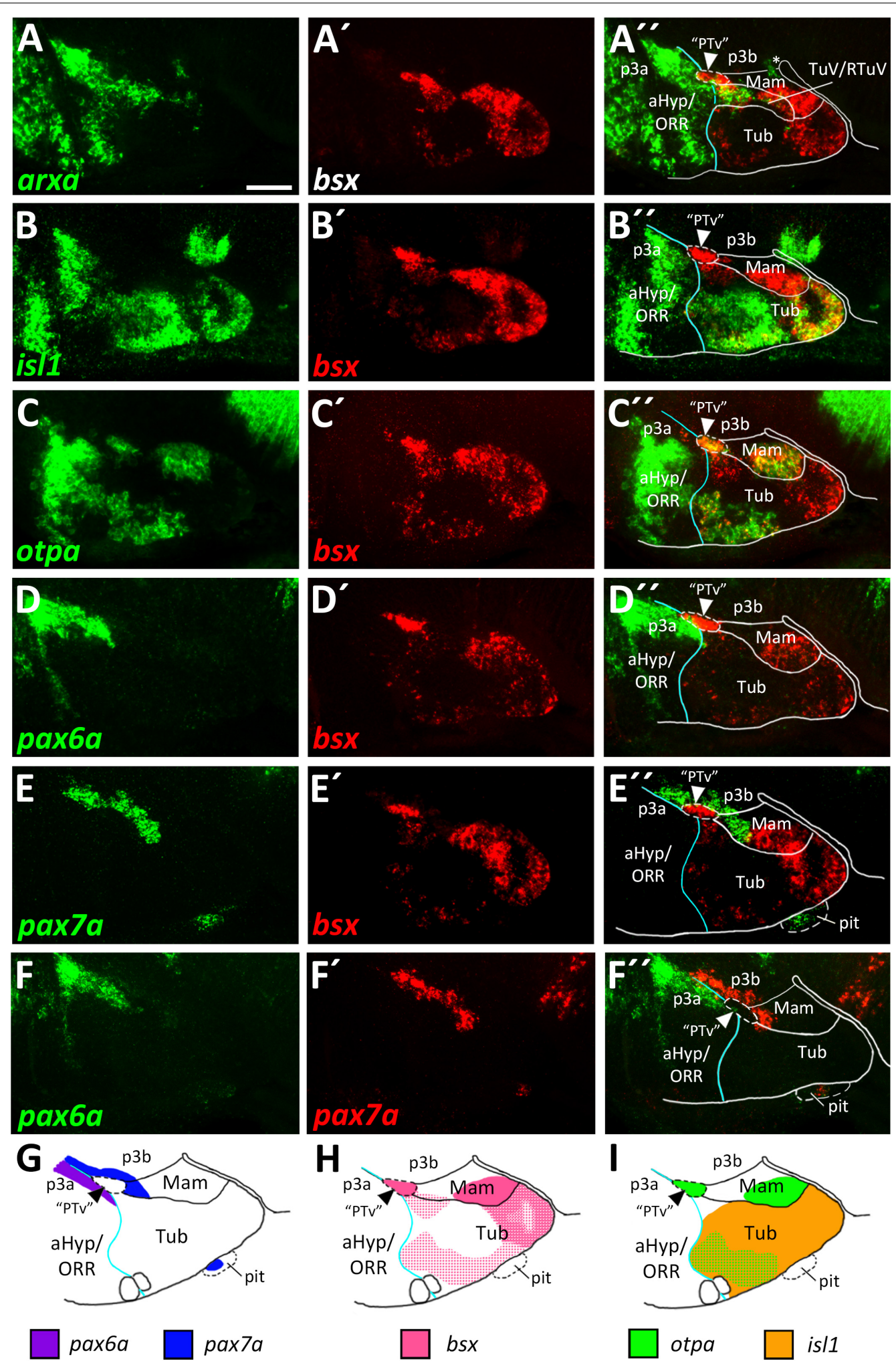

FIGURE 2 | bsx expression in relation to other patterning factors within the basal hypothalamus. (A-F') Sagittal optical sections of zebrafish embryos 48 hpf, stained by double-fluorescent whole-mount in situ hybridization using probes as indicated. Schematics show expression patterns of pax6a, pax7a (G), bsx (H), otpa and is/1 (I) in and close to the basal hypothalamus. Asterisk in $\left(\mathbf{A}^{\prime \prime}\right)$ indicates arxa expression in the floor plate. Arrowheads indicate the domain we operationally define as "PTv." The alar-basal boundary is shown in cyan. Maximum intensity projections of 20 (A-B", D-E'), 25 (F-F'), or 30 (C-C') $1 \mu \mathrm{m}$ confocal planes. Abbreviations see list. Scale bar $50 \mu \mathrm{m}$. 

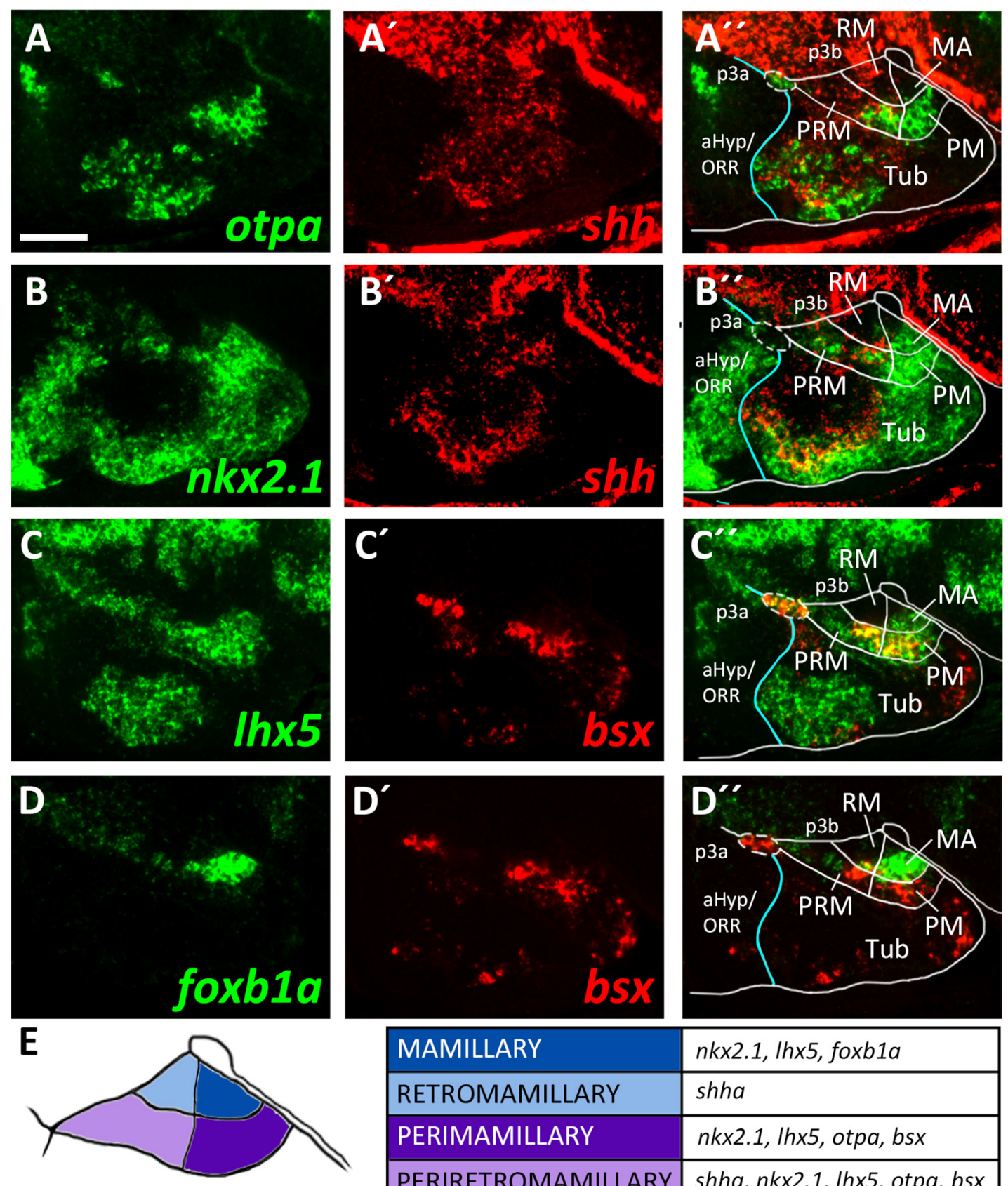

\begin{tabular}{|l|l|}
\hline MAMILLARY & $n k \times 2.1, I h \times 5$, foxb1a \\
\hline RETROMAMILLARY & $s h h a$ \\
\hline PERIMAMILLARY & $n k \times 2.1, \ln \times 5$, otpa, $b s x$ \\
\hline PERIRETROMAMILLARY & $s h h a, n k \times 2.1, \ln \times 5$, ot $p a, b s \times$ \\
\hline
\end{tabular}

FIGURE 3 | Longitudinal and transverse subdivisions of the mamillary regions based on conserved gene expression domains. (A-D') Sagittal optical sections of ventricular hypothalamic regions in zebrafish embryos $48 \mathrm{hpf}$, stained by double-fluorescent whole-mount in situ hybridization using probes as indicated.

(E) Schematic showing subdomains of the mamillary region and the genes expressed therein. The alar-basal boundary is shown in cyan. Maximum intensity

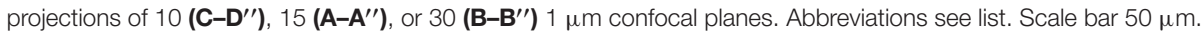

to the floorplate, i.e., embryologically more ventral. On the other hand, the foxbla expression domain, indicative for the MA, was considerably smaller in lateral areas (compare Figures 3D-D ${ }^{\prime \prime}$ and Supplementary Figures S4D-D'). Dorsal views confirmed the foxb1a expression domain to reach more

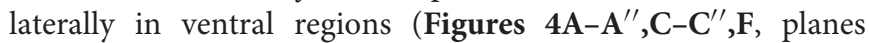
indicated in Figures $4 \mathrm{E}, \mathrm{H}$ ) and to be restricted to medial regions more dorsally (Figures $4 \mathbf{B}-\mathbf{B}^{\prime \prime}, \mathbf{D}-\mathbf{D}^{\prime \prime}, \mathbf{G}$, planes indicated in Figures $4 \mathbf{E}, \mathbf{H})$. Using otpa as a marker, we found the PM to surround the foxb1a expressing MA area in a cup-like shape (Figures 4C-D ${ }^{\prime \prime}, \mathbf{H}$ ). Consistent with this model and with our previous observations, we found $b s x$, which is expressed in both $\mathrm{PM}$ and tuberal regions, to be expressed as a broader band surrounding the MA (Figures $\mathbf{4 A - \mathbf { B } ^ { \prime \prime }}$ ). We summarized our 

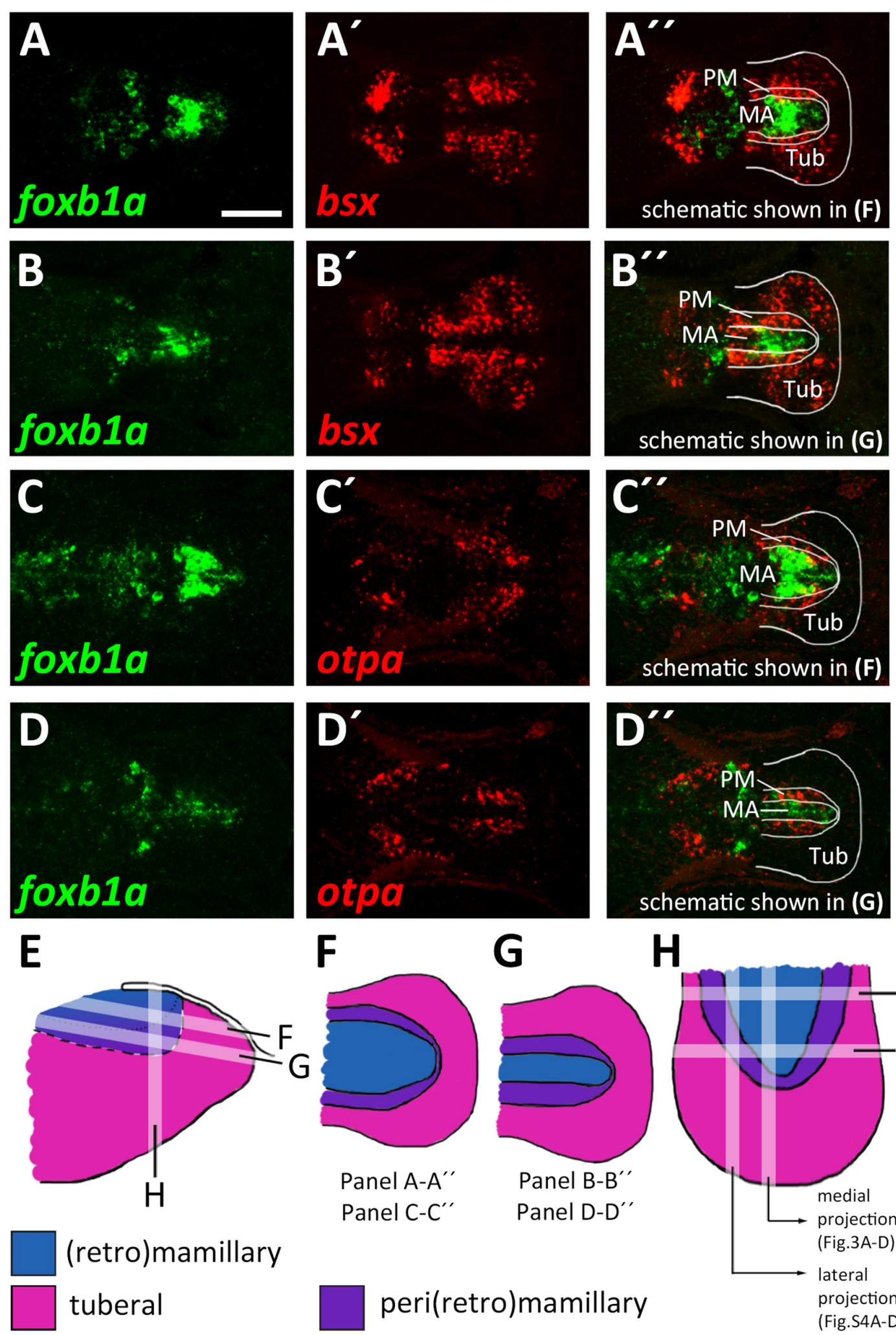

$\mathbf{F}$
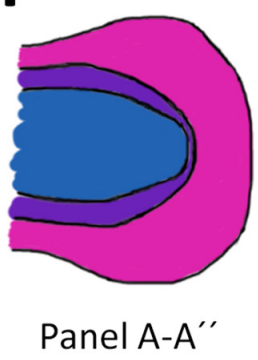

Panel C-C"

G

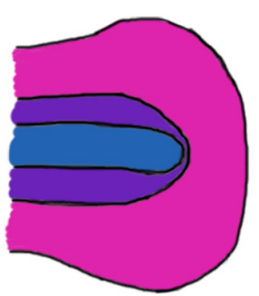

Panel B-B"

Panel D-D"
H

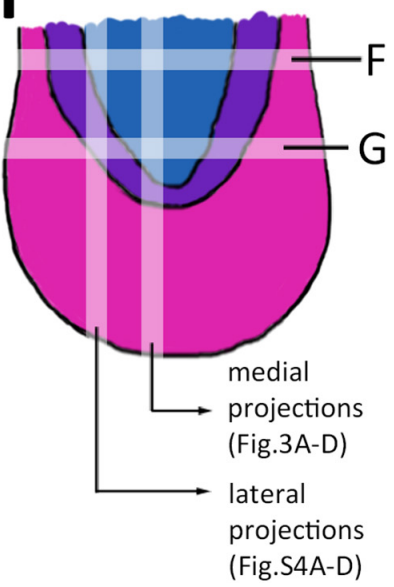

FIGURE 4 | Radial organization of the basal hypothalamus. (A-D") Horizontal optical sections of ventricular hypothalamic regions in zebrafish embryos 48 hpf, stained by double-fluorescent whole-mount in situ hybridization using probes as indicated. (E) Schematics showing lateral view on basal hypothalamus with tuberal and mamillary regions color coded as indicated. Section planes of schematics representing horizontal $\mathbf{( F , G ) ~ a n d ~ s a g i t t a l ~} \mathbf{( H )}$ projections are shown. Maximum intensity projections of $10\left(\mathbf{A}-\mathbf{B}^{\prime \prime}, \mathbf{D}-\mathbf{D}^{\prime \prime}\right)$ or $15\left(\mathbf{C}-\mathbf{C}^{\prime \prime}\right) 1 \mu \mathrm{m}$ confocal planes. Abbreviations see list. Scale bar $50 \mu \mathrm{m}$. 
model of the radial organization within this hypothalamic area in Figures 4E-H.

\section{The Zebrafish Larval Tuberal Hypothalamus Can Be Subdivided Into Longitudinal and Transverse Subterritories}

We already showed that in zebrafish an $a r x a^{+}, \operatorname{lh} x 6^{+}$band extends from alar regions into the basal hypothalamus (Figures 2A-A', Supplementary Figures S2B-J, S3A-A'). Following our aim to find the most parsimonious model in terms of homologous gene expression domains marking conserved progenitor domains in the basal hypothalamus, we considered this domain to correspond to RTuV/TuV (Figures $5 \mathbf{C}-\mathbf{C}^{\prime \prime}$ ). We examined tuberal shha expression, which has been shown to extend into terminal regions in $\mathrm{TuD}$ (Puelles et al., 2012) and in shell regions of the ventromedial hypothalamus area (VMH; Morales-Delgado et al., 2011).

In line with this, we observed a broad band of shha expression which reaches the acroterminal domain close to the alar-basal boundary (Figures $\mathbf{5} \mathbf{A}^{\prime}, \mathbf{B}$ ). In mouse, where this Shh expression domain is conserved, this area is named TuD or anterobasal region (ABas). Topologically ventral to this, shha expression was also observed in terminal regions but did not reach the acroterminal end of the forebrain. This domain, just like the $\mathrm{TuD} / \mathrm{ABas}$ domain, also expresses $n r 5 a 2$ (Figures $\mathbf{5} \mathbf{F}-\mathbf{F}^{\prime \prime}$ ). Since the hypothalamic expression domain of $n r 5 a 2$ has been demonstrated to overlap with nr5ala expression (Kurrasch et al., 2007), the mouse homolog of which has been described to be selectively expressed in the TuD/ABas and VMH regions around E13.5 (Puelles et al., 2012; Ferran et al., 2015), we operationally defined this region as VMH. The presence of penka expression (Figure 6C) supports our interpretation that this region shares homology with the mammalian VMH. In line with the literature (Morales-Delgado et al., 2011), we found that both the terminal $\mathrm{TuD} / \mathrm{ABas}$ and $\mathrm{VMH}$ domain are the only tuberal regions which are $d l x 5 a$ negative (Figure 5A). Furthermore, we found that the $n r 5 a 2^{+}, d l x 5 a^{-} \mathrm{TuD} / \mathrm{ABas}$ and $\mathrm{VMH}$ regions also expresses otpa (Figures 3A-A'). Otp expression in this tuberal subregion has previously been described in mammals (Morales-Delgado et al., 2011), amphibians and reptiles (Dominguez et al., 2015). The same area also expresses $l h x 5$ (Figures $3 \mathbf{C}-\mathbf{C}^{\prime \prime}$ ), which has previously been reported to be expressed in a homologous region in catshark (Santos-Duran et al., 2018).

In a large tuberal area embryologically ventral to this (the previously named "cHyp" area as indicated in Figure 1G), we found no shha expression (Figures $\mathbf{5} \mathbf{A}-\mathbf{B}^{\prime \prime}$ ). Absence of shha expression as well as presence of lef1 expression have previously been described for the remaining parts of the terminal intermediate tuberal (TuI) domain (excluding the VMH area; Ferran et al., 2015). Based on lef1 expression in this area (Figures 5D-D" and Supplementary Figures $\mathbf{S 5 E}-\mathrm{E}^{\prime \prime}$ ), we assume that this area shares some homology with mammalian TuI parts. However, we would like to note that this region in teleosts has features which are absent from the mammalian TuI region, most notably the posterior hypothalamic recess
(PR; Mueller and Wullimann, 2016; Xavier et al., 2017; Yamamoto et al., 2017). We hypothesize that the PR might cause the displacement of the $\ln x 6^{+} \mathrm{TuV}$ domain to far parasagittal regions (Supplementary Figure S6). Notably, the posterior recess region (PRR) also shows features characteristic for the $\mathrm{TuV}$ domain, i.e., the presence of histaminergic neurons at embryonic stages (Eriksson et al., 1998). To highlight our observation that this regions has tuberal (both $\mathrm{TuI}$ and $\mathrm{TuV}$ ) characteristics, we designated this area Tub/PRR.

We also detected a thin band of $f g f 8 a$ expression reaching from an embryologically anterior part of the PM area to an area just above the pituitary (Supplementary Figures S2B-J) and concluded that it represents parts of the acroterminal domain as has been suggested previously in mice (Ferran et al., 2015).

We found $\operatorname{lh} x 9$ expression in a peduncular dorsal tuberal domain close to the alar-basal boundary (Figures $5 \mathbf{E}-\mathbf{E}^{\prime \prime}$ ), similarly to what has previously been reported for mouse (Puelles et al., 2012). This region, in contrast to the terminal $\mathrm{TuD}$ parts, expresses $d l x 5 a$ (Figures $\mathbf{5} \mathbf{A}-\mathbf{A}^{\prime \prime}$ and Supplementary Figures $\left.\mathbf{S 5 A}-\mathbf{B}^{\prime \prime}, \mathbf{F}, \mathbf{F}^{\prime}\right)$ and not $n r 5 a 2\left(\right.$ Figures $\left.5 \mathbf{F}-\mathbf{F}^{\prime \prime}\right)$. Following the updated prosomeric model we thus distinguished between a peduncular $l h x 9^{+}, d l x 5 a^{+}, n r 5 a 2^{-}$RTuD domain and a terminal lh $x 9^{-}, d l x 5 a^{-}, n r 5 a 2^{+} \mathrm{TuD}$ domain.

We observed that the zebrafish RTuD and RTuI domains do not express $n k x 2.1$ (Figures 3B-B" and Supplementary Figures S5C-C ${ }^{\prime \prime}$ ), while in mouse $n k x 2.1$ expression was found in all tuberal subregions (Puelles et al., 2012). However, previous work from our group showed that both $n k x 2.4 a$ and $n k x 2.4 b$ are expressed in this region and that those genes act partially redundantly (Manoli and Driever, 2014), suggesting that $n k \times 2.4$ gene products substitute $n k \times 2.1$ gene product functions therein.

We found $n k \times 2.2 a$ expression along the alar-basal boundary but also in both $\mathrm{RTuD}$ and $\mathrm{TuD}$ (Figures $\mathbf{5 G}-\mathbf{G}^{\prime \prime}$ ). $N k \times 2.2 a$ expression in mice has been shown to be restricted to the alarbasal boundary regions in early stages but starting around E13.5 to expand into more ventral tuberal regions, contributing to both the developing VMH and peduncular dorsomedial hypothalamic nucleus (DMH; Puelles et al., 2012), a derivative of the RTuI domain. This reflects previous descriptions of $n k x 2.2 a$ expression in the zebrafish VMH (Kurrasch et al., 2007) and helped us to identify a border between the peduncular and terminal region within the intermediate tuberal domains. We concluded that $n k x 2.2 a$ is expressed in $\mathrm{RTuD}$ and RTuI as well as in the terminal $\mathrm{TuD} / \mathrm{AB} a$ s region and $\mathrm{VMH}$ region. The remaining (non-VMH) terminal Tub/PRR region, which expresses lef1 (Figures 5D-D"; Ferran et al., 2015), as well as an acroterminal domain beneath the VMH area, both do not express $n k x 2.2 a$ (Figures 5G-G' $\mathbf{G}^{\prime \prime}$ ).

We noted that the acroterminal $n k x 2.2 a^{-}$region is the same area which appears to be mainly shha negative. Based on this gene expression profile and on the location of this region right above the pituitary, we hypothesized that it might correspond to the developing arcuate nucleus (ARC). The ARC is anatomically often defined through expression of its hallmark neuropeptides. While it has been shown that Pomc expressing neurons actually originate in the $\mathrm{TuD} / \mathrm{ABas}$ region and only secondarily migrate into the ARC region (Díaz et al., 2014), 

Agrp expressing neurons have been described as to originate directly in the ARC (Díaz et al., 2014). We thus assessed agrp expression within the isl1 expressing tuberal hypothalamus at $48 \mathrm{hpf}$ (Figure 6A, arrowhead) and found several agrp ${ }^{+}$cells within this acroterminal region adjacent to the pituitary. At 96 hpf we found agrp expressing neurons spread over a longer part of the acroterminal domain (Figure 6B, arrowhead) and hypothesized this region to represent a structure homologous to the mammalian ARC, similarly to what has previously been suggested (Forlano and Cone, 2007). We also observed few agrp expressing cells within the $\mathrm{RTuD}$ region (Figures 6A,B, arrow), which, to our knowledge, were never observed in the mammalian hypothalamus and which might express agrp only transiently as they were not described in later larval stages (Shainer et al., 2017).

Our analysis allowed us to establish a map for the embryonic zebrafish basal hypothalamus, showing the subdivisions as defined within the prosomeric model (Figure 7A) and to describe the expression pattern of the $b s x$ gene within these regions (Figure $7 \mathbf{B}$ ). We found $b s x$ to be expressed in the tuberal Tub/PRR, ARC and TuD/ABas regions while the $\mathrm{VMH}$ area as well as the retrotuberal areas are mainly $b s x$ negative with the exception of some dispersed cells. In the perimamillary regions as well as in the posterior tubercular area containing dopaminergic "PTar" neurons, we found $b s x$ expression to perfectly overlap with otpa expression. We
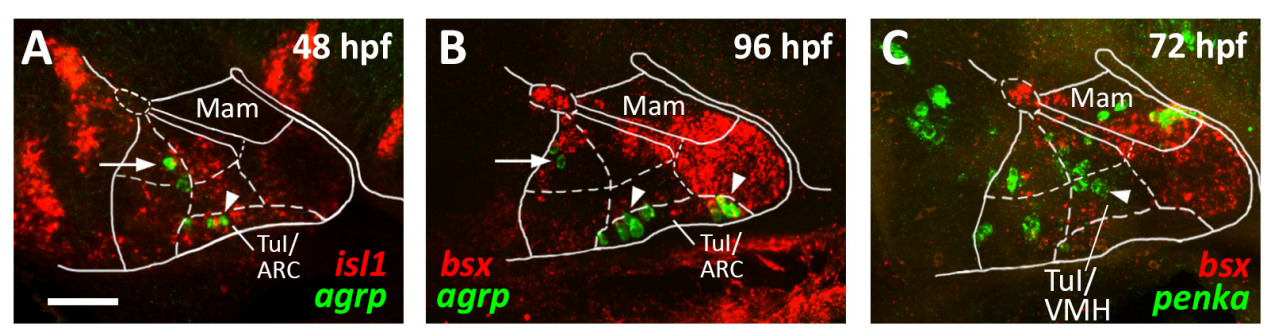

FIGURE 6 | agrp and penka expression in tuberal subregions. Sagittal optical sections of hypothalamic regions in zebrafish embryos 48 hpf (A), 96 hpf (B), or 72 hpf, stained by double-fluorescent whole-mount in situ hybridization using probes as indicated. White arrowheads in (A,B) indicate agrp ${ }^{+}$cells in the putative arcuate nucleus region; white arrows indicate agrp ${ }^{+}$neurons in retrotuberal territories. White arrowhead in (C) indicates penka+ cells in the putative ventromedial hypothalamus region. The alar-basal boundary is shown in cyan. Maximum intensity projections of 20 (A), 30 (B), or 50 (C) $1 \mu \mathrm{m}$ confocal planes. Abbreviations see list. Scale bar $50 \mu \mathrm{m}$.

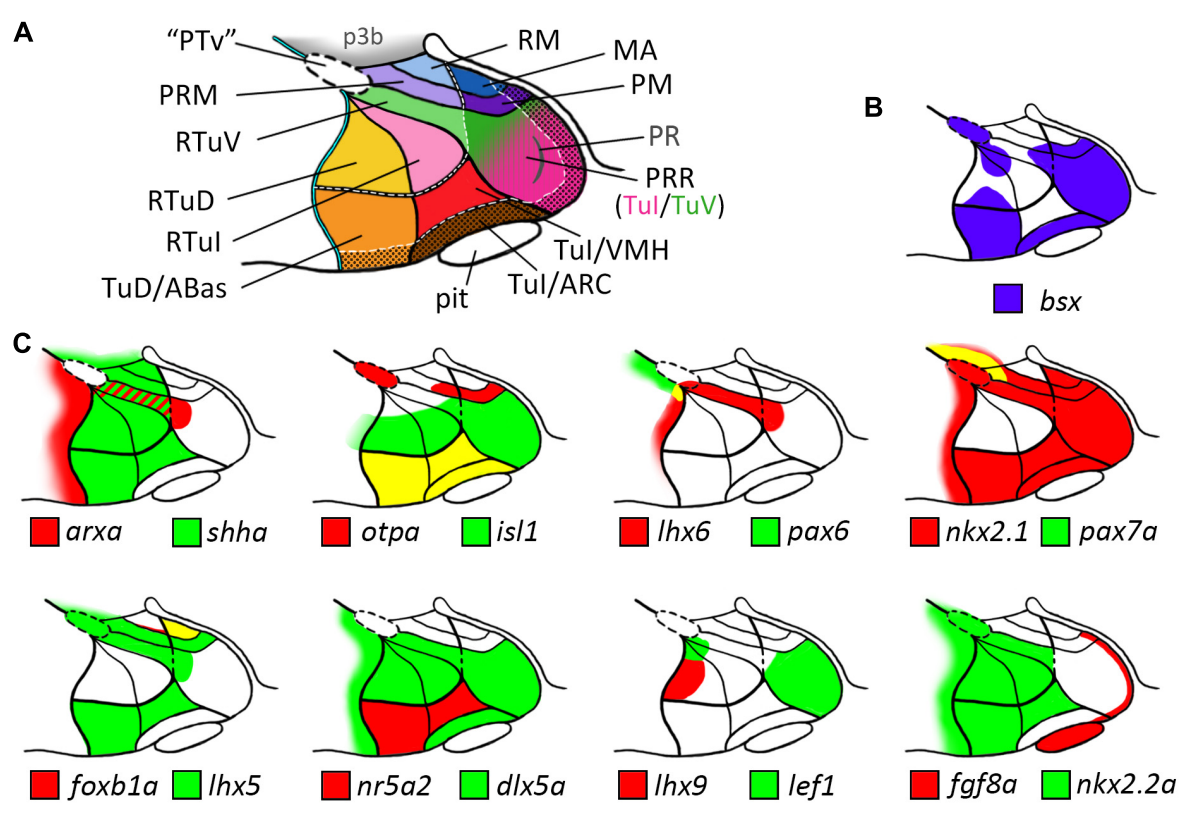

FIGURE 7 | Schematic representations of gene expression patterns in subdomains of the basal hypothalamus. (A) Schematic of a representative intermediate sagittal optical section highlighting all subdomains of the basal hypothalamus identified in this study. The alar-basal boundary is shown as thin cyan line with black border. White hatched lines indicate transverse boundaries between the peduncular and terminal hypothalamus as well as between the terminal (solid colors) and acroterminal (shown with black dotted overlay pattern) domains. The green/pink hatched gradient fill area indicates the mixed TuV-and Tul-like characteristics of the PRR (see main text for details). (B,C) Expression patterns of (B) bsx in purple and (C) other genes in red and green as indicated within the subdomains of the basal hypothalamus as depicted in (A), yellow indicates overlapping expression. The red-green hatched area in (C) indicates expression at different sagittal levels, thus no colocalization. Abbreviations see list. 
summarized the expression of all other analyzed genes within the basal hypothalamus in Figure 7C.

\section{DISCUSSION}

The extensive utilization of gene expression data has revolutionized our understanding of the evolutionary conserved genoarchitecture of the hypothalamus in mammals and other vertebrate classes, resulting in an updated prosomeric model of hypothalamic organization (Puelles et al., 2012; Puelles and Rubenstein, 2015). However, so far this highly successful approach has not been fully extended to teleosts, and specifically the zebrafish model, which is widely used by neurobiologists to study development and function of the hypothalamus (Löhr and Hammerschmidt, 2011; Xie and Dorsky, 2017). The updated prosomeric model of hypothalamic organization has largely been built upon data from the Allen Developing Mouse Brain Atlas $^{1}$, containing E11.5, E13.5, and E15.5 expression patterns of many genes involved in hypothalamic development. For both the embryonic and adult zebrafish brain several online atlas projects have been initiated by our group and others (Ronneberger et al., 2012; Randlett et al., 2015; Tabor et al., 2019). However, these anatomical gene expression frameworks are largely restricted to late embryonic and early larval stages, when most of the early patterning markers cease their broad expression and may be maintained only in a subset of differentiated neurons, making the assessment of genoarchitecture more difficult. Our experience with the VibeZ expression data registration and $48 \mathrm{hpf}$ anatomical reference framework (Ronneberger et al., 2012) revealed that proper anatomical registration in praxis is very sensitive to variations in cephalic flexure, which tend to generate artifacts during elastic registration especially in the mamillary and tuberal hypothalamus. Therefore, the classical neuroanatomical approach using pairwise mapping of gene expression domains by double fluorescent in situ hybridization was preferred in our study.

For analyzing the genoarchitecture of the embryonic basal hypothalamus, we chose $48 \mathrm{hpf}$ since at this stage the major morphogenetic reorganization of the embryonic CNS is accomplished, and local organizing centers have established regional identities in the brain (Mueller and Wullimann, 2016). Importantly, many transcription factor genes involved in pattern formation are still broadly expressed in their anatomical domains $48 \mathrm{hpf}$. While we did not compare different stages of zebrafish hypothalamus development extensively enough to hypothesize about a potential phylotypic stage, we found marker gene expression domains in most cases to be topologically equivalent in mouse E13.5 and zebrafish $48 \mathrm{hpf}$ brains. In general, our data revealed the genoarchitecture of the embryonic basal hypothalamus to be highly conserved between zebrafish and mouse, i.e., to consist of topologically equivalent subregions defined through homologous gene expression domains (Figure 7). It will be interesting to see how this conservation relates to the anatomical location of neuropeptidergic and neuromodulatory cell types, many of

${ }^{1} \mathrm{http} / / /$ developingmouse.brain-map.org which were recently mapped for the mouse model (Díaz et al., 2014). Proponents of the updated prosomeric model repeatedly pointed out that too little attention has been paid to the cephalic flexure, and to the resulting discrepancies in embryological terms of location (Puelles and Rubenstein, 2003; Puelles et al., 2012). We believe that this is particularly true for zebrafish. To illustrate this, we would like to use the PRR as an example. Neuroanatomical studies, published more than 20 years ago, already suggested this area to correspond to tuberal hypothalamus (Wullimann et al., 1996) but used the term caudal Hypothalamus to refer to the location of this area relative to the body axis and not the neuraxis. Synonymously, recent papers often use the term "posterior hypothalamus" to refer to this brain structure (Wolf and Ryu, 2013; Muthu et al., 2016). This nomenclature could lead to misconceptions as it might be associated with the posterior nucleus of the hypothalamus which is actually a derivative of the mamillary hypothalamus (Puelles et al., 2012). Based on its relative location to the anterior end of the floorplate, the expression of multiple genuinely tuberal markers, such as $d l x 5 a$ or isll, and the absence of mamillary markers, such as $\operatorname{lh} x 5$, we classified this region as tuberal.

We found the tuberal part of the PRR to be quite large and to overgrow parts of the mamillary hypothalamus. While also in mice the mamillary regions have been described to be broader in medial regions and thinner in lateral regions (Puelles et al., 2012; Puelles, 2019), the difference in size appears to be even more pronounced in zebrafish. It has been proposed by others to conceptualize the hypothalamus to consist of "modules," which are gained or lost during evolution (Xie and Dorsky, 2017). Tetrapods have only one hypothalamic recess (of the $3 \mathrm{rd}$ ventricle) which is thought to be homologous to the lateral recess in teleosts (Yamamoto et al., 2017). An additional posterior hypothalamic recess, however, is present in several bony (Parent and Northcutt, 1982; Kotrschal et al., 1985) and cartilaginous fish (Meurling and Rodríguez, 1990; Carrera et al., 2012; Rodríguez-Moldes et al., 2017). The PRR in zebrafish is densely populated by CSF-c neurons, many of which contain monoamines such as dopamine and serotonin (Xavier et al., 2017). In zebrafish, histaminergic neurons are located in the PRR (Eriksson et al., 1998) and were associated with a "posterior paraventricular organ" in the adult brain (Kaslin and Panula, 2001). In mammals, the histaminergic neurons are located in the premamillary nucleus (Panula et al., 1984) and were suggested to originate in the $\mathrm{RTuV} / \mathrm{TuV}$ region (Puelles et al., 2012). Notably, the RTuV/TuV has been associated with a hypothalamic ventricular organ, a "linear circumventricular specialization which is unremarkable in mammals" (Puelles and Rubenstein, 2015). The zebrafish PRR exhibits a high degree of continuous neurogenesis (Grandel et al., 2006; Wang et al., 2012). This might cause the overgrowth of the tuberal PRR and the peculiar shape of the $\mathrm{RTuV} / \mathrm{TuV}$ domain which could be explained through anisotropic growth (Fu et al., 2019) of tuberal regions as the PRR bulges outward. To account for its divergent phylogenetic status, we suggest for this brain region not to adopt the mammalian nomenclature but to refer to it as PRR.

An alternative or additional explanation for the unexpected shape of the $\mathrm{TuV}$ domain as inferred from lhx6 and arxa expression may be considered, which is a fundamental limit of the 
histogenetic markers used in this and other studies. Genes such as $\operatorname{lh} x 6$ and arxa are transiently expressed in multiple developing neurons during progenitor states and maintained in only a small subset of maturing neurons. It is thus possible that cells in the anterior parts of the ventral tuberal regions (Supplementary Figure S2J, gray hatched area labeled "hyp. TuV") ceased to express lhx6 and arxa or migrated to other regions.

A fundamental anatomical landmark, the alar-basal boundary, in 48 hpf zebrafish larvae has recently been proposed in a way that the shha expression within the secondary prosencephalon reaches into the alar plate (Affaticati et al., 2015; Vernier, 2017). We slightly revise this model to make it more parsimonious and propose the alar-basal boundary to coincide with the boundary between the shha and arxa expression domains, thus proposing the shha expression in the secondary prosencephalon to be entirely basal, similar to what has been suggested in mouse (Puelles et al., 2012) and catshark (Santos-Duran et al., 2015).

A more complicated boundary, in our eyes, is the boundary between the hypothalamus and the diencephalon, i.e., prosomer 3. This boundary region has been referred to as posterior tuberculum and has been of special interest because of dopaminergic cell populations, traditionally named diencephalic clusters (DCs), being located within or in proximity to this region (Rink and Wullimann, 2002). By comparing $b s x$ expression to the expression of mamillary markers such as $\operatorname{lh} x 5$ and otpa and also to the expression of $t h$, we were able to resolve the location of some of those dopaminergic cell clusters. Bs $x$ and Otp expression colocalization has previously been shown in mouse in a structure called "ventral mamillary body" (Cremona et al., 2004). Furthermore, colocalization of otpa and th expression within the mamillary regions has been demonstrated in Xenopus (Dominguez et al., 2014).

The DC2, 4, 5, and 6 clusters have been shown to be homologous to the A11 dopaminergic system based on their dependency on Otp (Ryu et al., 2007) and their projection pattern (Tay et al., 2011). Ryu et al. (2007) already demonstrated that in mice, A11 neurons are Nkx2.1 positive, despite many of them being localized in thalamic areas, arguing for a potential migration of progenitors from the hypothalamus to the thalamus. Mouse A11 neurons are distributed in the posterior and dorsal hypothalamic areas as well as in thalamic regions (Smeets and González, 2000). Based on our colocalization analysis we consider DC5 and 6 to be located within mamillary $b s x$ and otp $a$ expressing regions. It is tempting to speculate that DC5 and 6 might be homologous to A11 neurons of the hypothalamus, while DC2 and potentially also DC4 might correspond to A11 neurons which in mammals have been suggested to migrate into thalamic regions, thereby possibly crossing $\mathrm{p} 3 \mathrm{~b}$. In catshark, th expressing cells in a similar region of the brain have been interpreted as being located on both sides of the boundary between hypothalamic mamillary areas and p3b (Santos-Duran et al., 2015).

Based on the data we present here, we could not determine the exact location of the DC2 and DC4 dopaminergic neurons, which differentiate already at neural plate stages (Mahler et al., 2010). This is well before the complex genoarchitectural pattern which we described for the $48 \mathrm{hpf}$ zebrafish embryo is established. Most genoarchitectural markers are expressed in progenitor cells residing close to the ventricle, and their expression often does not extend into the far lateral mantle regions harboring mature DC2 and DC4 dopaminergic neurons. For the area in which DC2 neurons are located in mantle regions, we found $b s x$ expression more medially in intermediate sagittal optical sections. The anatomical status of this area, located both at the alar-basal boundary and at the diencephalic-hypothalamic border, remains challenging. To avoid premature conclusions about this area, we operationally labeled it with "PTv." However, further studies are needed and might suggest a hypothalamic, prethalamic ( $\mathrm{p} 3 \mathrm{a})$ or diencephalic tegmental (p3b) identity of this area. In fact, if the tegmental nature of this area will be refuted in future studies it should no longer be referred to as "posterior tuberculum," a structure which has been tightly associated with p3b (Mueller and Wullimann, 2016). We hope that the framework we generated in this study will provide a solid ground for future work in determining both origin and destination of DC2 and 4 dopaminergic cells, as well as the exact location of the hypothalamic-diencephalic boundary, which has long been and still is subject of lively discussion among zebrafish neuroanatomists (Biran et al., 2015; Yamamoto et al., 2017).

Genoarchitectonic maps have been worked out for the hypothalamus mainly based on expression data generated on mouse brain slices (Puelles et al., 2012; Díaz et al., 2014; Ferran et al., 2015). These maps provide great tools to facilitate understanding of a complex three-dimensional brain structure. Here, we have extended this model to the evolutionary distant zebrafish embryo, which is an excellent experimental system for the analysis of brain patterning mechanisms, but also for studies of neural circuit formation and function. The gross anatomical differences between fish and mammals have so far hindered the comparison of regulatory circuitries involving the hypothalamus. The emerging shared genoarchitecture of both systems will facilitate the transfer of insights into developmental mechanisms as well as neural circuitries.

\section{DATA AVAILABILITY STATEMENT}

All datasets generated for this study are included in the article/Supplementary Material.

\section{ETHICS STATEMENT}

The animal study was reviewed and approved by the Regierungspraesidium Freiburg.

\section{AUTHOR CONTRIBUTIONS}

TS conceptualized and designed the study and performed the experiments, assembled the figures, and wrote the first draft of the manuscript. WD contributed to design, supervision and editing, and provided project administration and funding acquisition.

\section{FUNDING}

This study was funded by the Deutsche Forschungsgemeinschaft (DFG, German Research Foundation) under Germany's 
Excellence Strategy - EXC-2189 - Project ID: 390939984 [Gefördert durch die Deutsche Forschungsgemeinschaft (DFG) im Rahmen der Exzellenzstrategie des Bundes und der Länder EXC-2189 - Projektnummer 390939984] and the Excellence Initiative of the German Federal and State Governments (BIOSS - EXC 294).

\section{ACKNOWLEDGMENTS}

We thank the Microscopy and Image Analysis Platform and the Life Imaging Center Freiburg for technical assistance with

\section{REFERENCES}

Affaticati, P., Yamamoto, K., Rizzi, B., Bureau, C., Peyrieras, N., Pasqualini, C., et al. (2015). Identification of the optic recess region as a morphogenetic entity in the zebrafish forebrain. Sci. Rep. 5:8738. doi: 10.1038/srep 08738

Alié, A., Devos, L., Torres-Paz, J., Prunier, L., Boulet, F., Blin, M., et al. (2018). Developmental evolution of the forebrain in cavefish, from natural variations in neuropeptides to behavior. eLife 7:e32808. doi: 10.7554/eLife. 32808

Bandín Carazo, S., Morona, R., Moreno, N., and González, A. (2013). Regional expression of Pax7 in the brain of Xenopus laevis during embryonic and larval development. Front. Neuroanat. 7:48. doi: 10.3389/fnana.2013. 00048

Bedont, J. L., Newman, E. A., and Blackshaw, S. (2015). Patterning, specification, and differentiation in the developing hypothalamus. Wiley Interdiscip. Rev. Dev. Biol. 4, 445-468. doi: 10.1002/wdev.187

Biran, J., Tahor, M., Wircer, E., and Levkowitz, G. (2015). Role of developmental factors in hypothalamic function. Front. Neuroanat. 9:47. doi: 10.3389/fnana. 2015.00047

Carrera, I., Anadón, R., and Rodríguez-Moldes, I. (2012). Development of tyrosine hydroxylase-immunoreactive cell populations and fiber pathways in the brain of the dogfish Scyliorhinus canicula: new perspectives on the evolution of the vertebrate catecholaminergic system. J. Comp. Neurol. 520, 3574-3603. doi: $10.1002 / \mathrm{cne} .23114$

Cho, G., Lim, Y., Cho, I.-T., Simonet, J. C., and Golden, J. A. (2014). Arx together with FoxA2, regulates Shh floor plate expression. Dev. Biol. 393, 137-148. doi: 10.1016/j.ydbio.2014.06.012

Cremona, M., Colombo, E., Andreazzoli, M., Cossu, G., and Broccoli, V. (2004). $\mathrm{Bsx}$, an evolutionary conserved brain specific homeoboX gene expressed in the septum, epiphysis, mammillary bodies and arcuate nucleus. Gene Expr. Patterns 4, 47-51. doi: 10.1016/s1567-133x(03)00151-0

Díaz, C., Morales-Delgado, N., and Puelles, L. (2014). Ontogenesis of peptidergic neurons within the genoarchitectonic map of the mouse hypothalamus. Front. Neuroanat. 8:162. doi: 10.3389/fnana.2014.00162

Dominguez, L., Gonzalez, A., and Moreno, N. (2014). Characterization of the hypothalamus of Xenopus laevis during development. II. The basal regions. J. Comp. Neurol. 522, 1102-1131. doi: 10.1002/cne. 23471

Dominguez, L., Gonzalez, A., and Moreno, N. (2015). Patterns of hypothalamic regionalization in amphibians and reptiles: common traits revealed by a genoarchitectonic approach. Front. Neuroanat. 9:3. doi: 10.3389/fnana.2015. 00003

Dominguez, L., Morona, R., Gonzalez, A., and Moreno, N. (2013). Characterization of the hypothalamus of Xenopus laevis during development. I. The alar regions. J. Comp. Neurol. 521, 725-759. doi: 10.1002/cne.2 3222

Eriksson, K. S., Peitsaro, N., Karlstedt, K., Kaslin, J., and Panula, P. (1998). Development of the histaminergic neurons and expression of histidine decarboxylase mRNA in the zebrafish brain in the absence of all peripheral histaminergic systems. Eur. J. Neurosci. 10, 3799-3812. doi: 10.1046/j.14609568.1998.00394.x imaging and Daniel Armbruster and Nadja Bischoff for technical assistance in the staining procedures. We thank Philippe Vernier for critical discussions. We thank Matthias Hammerschmidt and Heiko Löhr for providing probes, Eva Carl for cloning the lhx6 plasmid and Sabine Götter for excellent fish care.

\section{SUPPLEMENTARY MATERIAL}

The Supplementary Material for this article can be found online at: https://www.frontiersin.org/articles/10.3389/fnana. 2020.00003/full\#supplementary-material

Ferran, J. L., Puelles, L., and Rubenstein, J. L. (2015). Molecular codes defining rostrocaudal domains in the embryonic mouse hypothalamus. Front. Neuroanat. 9:46. doi: 10.3389/fnana.2015.00046

Filippi, A., Durr, K., Ryu, S., Willaredt, M., Holzschuh, J., and Driever, W. (2007). Expression and function of nr4a2, lmx1b, and pitx3 in zebrafish dopaminergic and noradrenergic neuronal development. BMC Dev. Biol. 7:135. doi: 10.1186/ 1471-213X-7-135

Forlano, P. M., and Cone, R. D. (2007). Conserved neurochemical pathways involved in hypothalamic control of energy homeostasis. J. Comp. Neurol. 505, 235-248. doi: 10.1002/cne.21447

Fu, T., Pearson, C., Towers, M., and Placzek, M. (2019). Development of the basal hypothalamus through anisotropic growth. J. Neuroendocrinol. 31:e12727. doi: 10.1111/jne.12727

González, A., López, J. M., Morona, R., and Moreno, N. (2017). "1.08 the organization of the central nervous system of amphibians," in Evolution of Nervous Systems (Second Edition), ed. J. H. Kaas, (Oxford: Academic Press), 141-170. doi: 10.1016/b978-0-12-804042-3.00005-1

Grandel, H., Kaslin, J., Ganz, J., Wenzel, I., and Brand, M. (2006). Neural stem cells and neurogenesis in the adult zebrafish brain: origin, proliferation dynamics, migration and cell fate. Dev. Biol. 295, 263-277. doi: 10.1016/j.ydbio.2006. 03.040

Herget, U., Wolf, A., Wullimann, M. F., and Ryu, S. (2014). Molecular neuroanatomy and chemoarchitecture of the neurosecretory preoptichypothalamic area in zebrafish larvae. J. Comp. Neurol. 522, 1542-1564. doi: $10.1002 /$ cne. 23480

Joven, A., Morona, R., González, A., and Moreno, N. (2013). Spatiotemporal patterns of Pax3, Pax6, and Pax7 expression in the developing brain of a Urodele Amphibian, Pleurodeles waltl. J. Comp. Neurol. 521, 3913-3953. doi: 10.1002/ cne. 23385

Kaslin, J., and Panula, P. (2001). Comparative anatomy of the histaminergic and other aminergic systems in zebrafish (Danio rerio). J. Comp. Neurol. 440, 342-377. doi: 10.1002/cne.1390

Kimmel, C. B., Ballard, W. W., Kimmel, S. R., Ullmann, B., and Schilling, T. F. (1995). Stages of embryonic development of the zebrafish. Dev. Dyn. 203, 253-310. doi: 10.1002/aja.1002030302

Kotrschal, K., Krautgartner, W. D., and Adam, H. (1985). Distribution of aminergic neurons in the brain of the sterlet, Acipenser ruthenus (Chondrostei, Actinopterygii). J. Hirnforsch. 26, 65-72.

Kurrasch, D. M., Cheung, C. C., Lee, F. Y., Tran, P. V., Hata, K., and Ingraham, H. A. (2007). The neonatal ventromedial hypothalamus transcriptome reveals novel markers with spatially distinct patterning. J. Neurosci. 27, 13624-13634. doi: 10.1523/jneurosci.2858-07.2007

Löhr, H., and Hammerschmidt, M. (2011). Zebrafish in endocrine systems: recent advances and implications for human disease. Annu. Rev. Physiol. 73, 183-211. doi: 10.1146/annurev-physiol-012110-142320

Mahler, J., Filippi, A., and Driever, W. (2010). DeltaA/DeltaD regulate multiple and temporally distinct phases of notch signaling during dopaminergic neurogenesis in zebrafish. J. Neurosci. 30, 16621-16635. doi: 10.1523/ JNEUROSCI.4769-10.2010

Manoli, M., and Driever, W. (2014). nkx2.1 and $n k x 2.4$ genes function partially redundant during development of the zebrafish hypothalamus, preoptic region, and pallidum. Front. Neuroanat. 8:145. doi: 10.3389/fnana.2014.00145 
Martinez-Ferre, A., and Martinez, S. (2012). Molecular regionalization of the diencephalon. Front. Neuroanat. 6:73. doi: 10.3389/fnins.2012. 00073

Meurling, P., and Rodríguez, E. M. (1990). The paraventricular and posterior recess organs of elasmobranchs: a system of cerebrospinal fluid-contacting neurons containing immunoreactive serotonin and somatostatin. Cell Tissue Res. 259, 463-473. doi: 10.1007/bf01740772

Morales-Delgado, N., Castro-Robles, B., Ferrán, J. L., Martinez-de-la-Torre, M., Puelles, L., and Díaz, C. (2014). Regionalized differentiation of CRH, TRH, and GHRH peptidergic neurons in the mouse hypothalamus. Brain Struct. Funct. 219, 1083-1111. doi: 10.1007/s00429-013-0554-2

Morales-Delgado, N., Merchan, P., Bardet, S. M., Ferrán, J. L., Puelles, L., and Díaz, C. (2011). Topography of somatostatin gene expression relative to molecular progenitor domains during ontogeny of the mouse hypothalamus. Front. Neuroanat. 5:10. doi: 10.3389/fnana.2011.00010

Moreno, N., Dominguez, L., Morona, R., and González, A. (2012). Subdivisions of the turtle Pseudemys scripta hypothalamus based on the expression of regulatory genes and neuronal markers. J. Comp. Neurol. 520, 453-478. doi: $10.1002 /$ cne. 22762

Moreno, N., Joven, A., Morona, R., Bandin, S., Lopez, J. M., and Gonzalez, A. (2014). Conserved localization of Pax6 and Pax7 transcripts in the brain of representatives of sarcopterygian vertebrates during development supports homologous brain regionalization. Front. Neuroanat. 8:75. doi: 10.3389/fnana. 2014.00075

Moreno, N., Morona, R., López, J. M., and González, A. (2017). “1.22 - the diencephalon and hypothalamus of nonmammalian vertebrates: evolutionary and developmental traits," in Evolution of Nervous Systems (Second Edition), ed. J. H. Kaas, (Oxford: Academic Press), 409-426. doi: 10.1016/b978-0-12804042-3.00017-8

Mueller, T., and Wullimann, M. F. (2016). Atlas of Early Zebrafish Brain Development. Amsterdam: Elsevier Academic Press.

Muthu, V., Eachus, H., Ellis, P., Brown, S., and Placzek, M. (2016). Rx3 and Shh direct anisotropic growth and specification in the zebrafish tuberal/anterior hypothalamus. Development 143, 2651-2663. doi: 10.1242/dev.13 8305

Nagpal, J., Herget, U., Choi, M. K., and Ryu, S. (2018). Anatomy, development, and plasticity of the neurosecretory hypothalamus in zebrafish. Cell Tissue Res. 375, 5-22. doi: 10.1007/s00441-018-2900-4

Panula, P., Yang, H. Y., and Costa, E. (1984). Histamine-containing neurons in the rat hypothalamus. Proc. Natl. Acad. Sci. U.S.A. 81, 2572-2576. doi: 10.1073/ pnas.81.8.2572

Parent, A., and Northcutt, R. G. (1982). The monoamine-containing neurons in the brain of the garfish, Lepisosteus osseus. Brain Res. Bull. 9, 189-204. doi: 10.1016/0361-9230(82)90132-0

Puelles, L. (2019). Survey of midbrain, diencephalon, and hypothalamus neuroanatomic terms whose prosomeric definition conflicts with columnar tradition. Front. Neuroanat. 13:20. doi: 10.3389/fnana.2019.00020

Puelles, L., and Ferran, J. L. (2012). Concept of neural genoarchitecture and its genomic fundament. Front. Neuroanat. 6:47. doi: 10.3389/fnana.2012. 00047

Puelles, L., Martinez-de-la-Torre, M., Bardet, S., and Rubenstein, J. L. R. (2012). "Chapter 8 - Hypothalamus," in The Mouse Nervous System, eds C. Watson, G. Paxinos, and L. Puelles, (San Diego: Academic Press), 221-312.

Puelles, L., and Rubenstein, J. L. (2015). A new scenario of hypothalamic organization: rationale of new hypotheses introduced in the updated prosomeric model. Front. Neuroanat. 9:27. doi: 10.3389/fnana.2015.00027

Puelles, L., and Rubenstein, J. L. R. (2003). Forebrain gene expression domains and the evolving prosomeric model. Trends Neurosci. 26, 469-476. doi: 10.1016/ s0166-2236(03)00234-0

Randlett, O., Wee, C. L., Naumann, E. A., Nnaemeka, O., Schoppik, D., Fitzgerald, J. E., et al. (2015). Whole-brain activity mapping onto a zebrafish brain atlas. Nat. Methods 12, 1039-1046. doi: 10.1038/nmeth.3581

Reinig, S., Driever, W., and Arrenberg, A. (2017). The descending diencephalic dopamine system is tuned to sensory stimuli. Curr. Biol. 27, 318-333. doi: 10.1016/j.cub.2016.11.059
Rink, E., and Wullimann, M. F. (2002). Development of the catecholaminergic system in the early zebrafish brain: an immunohistochemical study. Dev. Brain Res. 137, 89-100. doi: 10.1016/s0165-3806(02)00354-1

Rodríguez-Moldes, I., Santos-Durán, G. N., Pose-Méndez, S., Quintana-Urzainqui, I., and Candal, E. (2017). "1.05 - the brains of cartilaginous fishes," in Evolution of Nervous Systems (Second Edition), ed. J. H. Kaas, (Oxford: Academic Press), 77-97. doi: 10.1016/b978-0-12-804042-3.00003-8

Ronneberger, O., Liu, K., Rath, M., Rue $\beta$, D., Mueller, T., Skibbe, H., et al. (2012). ViBE-Z: a framework for 3D virtual colocalization analysis in zebrafish larval brains. Nat. Methods 9, 735-742. doi: 10.1038/nmeth.2076

Ryu, S., Mahler, J., Acampora, D., Holzschuh, J., Erhardt, S., Omodei, D., et al. (2007). Orthopedia homeodomain protein is essential for diencephalic dopaminergic neuron development. Curr. Biol. 17, 873-880. doi: 10.1016/j.cub. 2007.04.003

Santos-Duran, G. N., Ferreiro-Galve, S., Menuet, A., Mazan, S., Rodriguez-Moldes, I., and Candal, E. (2018). The shark basal hypothalamus: molecular prosomeric subdivisions and evolutionary trends. Front. Neuroanat. 12:17. doi: 10.3389/ fnana.2018.00017

Santos-Durán, G. N., Ferreiro-Galve, S., Menuet, A., Quintana-Urzainqui, I., Mazan, S., Rodríguez-Moldes, I., et al. (2016). The shark alar hypothalamus: molecular characterization of prosomeric subdivisions and evolutionary trends. Front. Neuroanat. 10:113. doi: 10.3389/fnana.2016.00113

Santos-Duran, G. N., Menuet, A., Lagadec, R., Mayeur, H., Ferreiro-Galve, S., Mazan, S., et al. (2015). Prosomeric organization of the hypothalamus in an elasmobranch, the catshark Scyliorhinus canicula. Front. Neuroanat. 9:37. doi: 10.3389/fnana.2015.00037

Schredelseker, T., and Driever, W. (2018). Bsx controls pineal complex development. Development 145:dev163477. doi: 10.1242/dev.16 3477

Shainer, I., Buchshtab, A., Hawkins, T. A., Wilson, S. W., Cone, R. D., and Gothilf, Y. (2017). Novel hypophysiotropic AgRP2 neurons and pineal cells revealed by BAC transgenesis in zebrafish. Sci. Rep. 7:44777. doi: 10.1038/srep44777

Shimogori, T., Lee, D. A., Miranda-Angulo, A., Yang, Y., Wang, H., Jiang, L., et al. (2010). A genomic atlas of mouse hypothalamic development. Nat. Neurosci. 13, 767-775. doi: 10.1038/nn.2545

Smeets, W. J. A. J., and González, A. (2000). Catecholamine systems in the brain of vertebrates: new perspectives through a comparative approach. Brain Res. Rev. 33, 308-379. doi: 10.1016/s0165-0173(00)00034-5

Tabor, K. M., Marquart, G. D., Hurt, C., Smith, T. S., Geoca, A. K., Bhandiwad, A. A., et al. (2019). Brain-wide cellular resolution imaging of Cre transgenic zebrafish lines for functional circuit-mapping. eLife 8:e42687. doi: 10.7554/ eLife. 42687

Tay, T. L., Ronneberger, O., Ryu, S., Nitschke, R., and Driever, W. (2011). Comprehensive catecholaminergic projectome analysis reveals single-neuron integration of zebrafish ascending and descending dopaminergic systems. Nat. Commun. 2:171.

Vernier, P. (2017). "1.04 - the brains of teleost fishes," in Evolution of Nervous Systems (Second Edition), ed. J. H. Kaas, (Oxford: Academic Press), 59-75. doi: 10.1016/b978-0-12-804042-3.00004-x

Vernier, P., and Wullimann, M. F. (2009). "Evolution of the posterior tuberculum and preglomerular nuclear complex," in Encyclopedia of Neuroscience, eds M. D. Binder, N. Hirokawa, and U. Windhorst, (Heidelberg: Springer), 1404-1413. doi: 10.1007/978-3-540-29678-2_3167

Wang, X., Kopinke, D., Lin, J., McPherson, A. D., Duncan, R. N., Otsuna, H., et al. (2012). Wnt signaling regulates postembryonic hypothalamic progenitor differentiation. Dev. Cell 23, 624-636. doi: 10.1016/j.devcel.2012. 07.012

Wolf, A., and Ryu, S. (2013). Specification of posterior hypothalamic neurons requires coordinated activities of Fezf2, Otp, Sim1a and Foxb1.2. Development 140, 1762-1773. doi: 10.1242/dev.085357

Wullimann, M. F., Rupp, B., and Reichert, H. (1996). Neuroanatomy of the Zebrafish Brain: A Topological Atlas. Basel: Birkhäuser Verlag.

Xavier, A. L., Fontaine, R., Bloch, S., Affaticati, P., Jenett, A., Demarque, M., et al. (2017). Comparative analysis of monoaminergic cerebrospinal fluid-contacting cells in Osteichthyes (bony vertebrates). J. Comp. Neurol. 525, 2265-2283. doi: $10.1002 /$ cne. 24204 
Xie, Y., and Dorsky, R. I. (2017). Development of the hypothalamus: conservation, modification and innovation. Development 144, 1588-1599. doi: 10.1242/dev. 139055

Yamamoto, K., Bloch, S., and Vernier, P. (2017). New perspective on the regionalization of the anterior forebrain in Osteichthyes. Dev. Growth Diff. 59, 175-187. doi: $10.1111 /$ dgd. 12348

Zhao, T., Szabó, N., Ma, J., Luo, L., Zhou, X., and Alvarez-Bolado, G. (2008). Genetic mapping of Foxb1-cell lineage shows migration from caudal diencephalon to telencephalon and lateral hypothalamus. Eur. J. Neurosci. 28, 1941-1955. doi: 10.1111/j.1460-9568.2008.06503.x
Conflict of Interest: The authors declare that the research was conducted in the absence of any commercial or financial relationships that could be construed as a potential conflict of interest.

Copyright (c) 2020 Schredelseker and Driever. This is an open-access article distributed under the terms of the Creative Commons Attribution License (CC BY). The use, distribution or reproduction in other forums is permitted, provided the original author(s) and the copyright owner(s) are credited and that the original publication in this journal is cited, in accordance with accepted academic practice. No use, distribution or reproduction is permitted which does not comply with these terms. 